\title{
Inner-Resonance Conditions for Honeycomb Paperboard Cushioning Packaging System with Critical Component
}

\author{
Jun Wang, ${ }^{1}$ Zhi-geng Fan, ${ }^{2}$ Xiang Hong, ${ }^{1}$ and Li-xin Lu ${ }^{1}$ \\ ${ }^{1}$ Jiangsu Province Key Laboratory of Advanced Food Manufacturing Equipment and Technology, \\ Department of Packaging Engineering, Jiangnan University, Wuxi 214122, China \\ ${ }^{2}$ School of Light Industry, Zhejiang University of Science and Technology, \\ Hangzhou 310023, China
}

Correspondence should be addressed to Jun Wang; wangj_1982@jiangnan.edu.cn

Received 25 March 2013; Accepted 3 August 2013

Academic Editor: Changfeng Ge

Copyright (C) 2013 Jun Wang et al. This is an open access article distributed under the Creative Commons Attribution License, which permits unrestricted use, distribution, and reproduction in any medium, provided the original work is properly cited.

A dynamic model was proposed for a honeycomb paperboard cushioning packaging system with critical component. Then the coupled equations of the system were solved by the variational iteration method, from which the conditions for inner-resonance were obtained, which should be avoided in the cushioning packaging design.

\section{Introduction}

Honeycomb paperboard is widely applicated in packaging industry due to its excellent performance in energy absorption and vibration attenuation. During the past decades, the dynamic behaviors of honeycomb paperboard under dynamical compression and impact loading are studied thoroughly. Experimental studies, theoretical modeling, and numerical simulations are all involved [1-3]. However, the oscillation in the honeycomb paperboard packaging system is of inherent nonlinearity $[4,5]$, and it should be treated as a double-degree-of-freedom system when the critical component should be considered [6]. It is desirable to obtain the inner-resonance conditions for a coupled packaging system since the packaged product will be damaged even at a very low dropping height for packaged product with critical component [7]. However, it remains a problem to obtain the resonance condition for nonlinear packaging system, especially for multidegree-of-freedom nonlinear cushioning packaging system [7]. The variational iteration method (VIM) has been extensively used in various nonlinear sciences, the nonsmooth problem [8], the $q$-difference and the $q$-fractional equations [9-11], the fractional calculus [12, 13], the fuzzy equation [14], and many other nonlinear problems $[15,16]$.

The governing equations of the honeycomb paperboard cushioning packaging system with critical component can be expressed as [17]

$$
\begin{gathered}
m_{1} \frac{d^{2} x}{d t^{2}}+k_{1}(x-y)=0, \quad x(0)=0, \quad \dot{x}(0)=\sqrt{2 g h}, \\
m_{2} \frac{d^{2} y}{d t^{2}}+\sum_{i=1}^{9} a_{i} y^{i}-k_{1}(x-y)=0, \\
y(0)=0, \quad \dot{y}(0)=\sqrt{2 g h .}
\end{gathered}
$$

Here the coefficients $m_{1}$ and $m_{2}$ denote, respectively, the mass of the critical component, and the main part of product, $g$ defines the gravity, while $\beta_{i}$ represents the nonlinear elastic coefficient of honeycomb paperboard cushioning pad. $k_{1}$ is the coupling stiffness of the critical component, and $h$ is the dropping height.

By introducing these parameters $T_{0}=\sqrt{m_{2} / a_{1}}, L=$ $a_{1} / a_{2}$ and letting $X=x / L, Y=y / L, T=t / T_{0}$, and 
$\gamma_{i}=a_{i} a_{1}^{i-2} / a_{2}^{i-1}(i=3, \ldots, 9),(1)$ can be equivalently written in the following forms:

$$
\begin{gathered}
\ddot{X}+\omega_{01}^{2} X-\omega_{01}^{2} Y=0, \quad X(0)=0, \quad X^{\prime}(0)=\frac{T_{0}}{L} \sqrt{2 g h}, \\
\ddot{Y}+\omega_{02}^{2} Y+Y^{2}+\sum_{i=3}^{9} \gamma_{i} Y^{i}+\left(1-\omega_{02}^{2}\right) X=0, \\
Y(0)=0, \quad Y^{\prime}(0)=\frac{T_{0}}{L} \sqrt{2 g h,}
\end{gathered}
$$

where

$$
\begin{aligned}
\omega_{01} & =\lambda_{1}, \\
\omega_{02} & =\sqrt{1+\lambda_{1}^{2} \lambda_{2}}, \\
\lambda_{1} & =\frac{\omega_{1}}{\omega_{2}}, \\
\lambda_{2} & =\frac{m_{1}}{m_{2}}, \\
\omega_{1} & =\sqrt{\frac{k_{1}}{m_{1}}}, \\
\omega_{2} & =\sqrt{\frac{k_{2}}{m_{2}}} .
\end{aligned}
$$

\section{Variational Iteration Method}

The variational iteration method, VIM [18], first proposed by $\mathrm{He}$, has been widely applicated in solving many different kinds of nonlinear equations $[19,20]$. Applying the variational iteration method [8], the following iteration formulae can be constructed:

$$
\begin{aligned}
X_{1}= & X_{0}+\frac{1}{\omega_{01}} \int_{0}^{t} \sin \omega_{01}(s-t)\left\{\ddot{X}_{0}+\omega_{01}^{2} X_{0}-\omega_{01}^{2} Y_{1}\right\} d s, \\
Y_{1}= & Y_{0}+\frac{1}{\omega_{02}} \\
& \times \int_{0}^{t} \sin \omega_{02}(s-t)\left\{\ddot{Y}_{0}+\omega_{02}^{2} Y_{0}+Y_{0}^{2}+\sum_{i=3}^{9} \gamma_{i} Y_{0}^{i}\right\} d s .
\end{aligned}
$$

Beginning with the initial solutions

$$
\begin{aligned}
& X_{0}=A_{1} \sin \left(\Omega_{1} t\right), \\
& Y_{0}=A_{2} \sin \left(\Omega_{2} t\right),
\end{aligned}
$$

we have

$$
\begin{gathered}
Y_{1}=\sum_{i=1}^{9}\left\{p\left[\frac{K_{i}}{\omega_{02}\left(i^{2} \Omega_{2}^{2}-\omega_{02}^{2}\right)}\left(\omega_{02} \sin i \Omega_{2} \tau-i \Omega_{2} \sin \omega_{02} \tau\right)\right]\right. \\
\left.+q\left[\frac{K_{i}}{i^{2} \Omega_{2}^{2}-\omega_{02}^{2}}\left(\cos i \Omega_{2} \tau-\cos \omega_{02} \tau\right)\right]\right\}
\end{gathered}
$$$$
+A_{2} \sin \Omega_{2} \tau
$$

Substituting (6) into (2) yields

$$
\begin{aligned}
& X_{1}=\left(\omega_{01}^{2}-A_{1} \Omega_{1}^{2}\right) \frac{\omega_{01} \sin \Omega_{1} \tau-\Omega_{1} \sin \omega_{01} \tau}{\omega_{01}\left(\Omega_{1}^{2}-\omega_{01}^{2}\right)} \\
&+\zeta_{1} \lambda_{2}^{-2} A_{1} \Omega_{1} \frac{\cos \Omega_{1} \tau-\cos \omega_{01} \tau}{\Omega_{1}^{2}-\omega_{01}^{2}} \\
&-\omega_{01}^{2} p \sum_{i=1}^{9}\left\{\frac{K_{i}}{\omega_{02}\left(i^{2} \Omega_{2}^{2}-\omega_{02}^{2}\right)}\right. \\
& \cdot\left[\frac{i \Omega_{2}}{\omega_{01}} \cdot \frac{\omega_{01} \sin \omega_{02} \tau-\omega_{02} \sin \omega_{01} \tau}{\omega_{01}^{2}-\omega_{02}^{2}}\right. \\
&-\left.\left.-\frac{\omega_{02}\left(\omega_{01} \sin i \Omega_{2} \tau-i \Omega_{2} \sin \omega_{01} \tau\right)}{\omega_{01}\left(\omega_{01}^{2}-i^{2} \Omega_{2}^{2}\right)}\right]\right\} \\
& \sum_{i=1}^{9}\left[\frac { K _ { i } } { i ^ { 2 } \Omega _ { 0 2 } ^ { 2 } - \omega _ { 0 2 } ^ { 2 } } \cdot \left(\frac{\cos \omega_{01} \tau-\cos i \Omega_{2} \tau}{\omega_{01}^{2}-i^{2} \Omega_{2}^{2}}\right.\right. \\
&\left.\left.-\omega_{01}^{2} A_{2} \frac{\omega_{01} \operatorname{sos} \omega_{01} \tau-\cos \omega_{02} \tau}{\omega_{01}^{2}-\omega_{02}^{2}}\right)\right] \\
& \omega_{01}\left(\Omega_{2}^{2}-\omega_{01}^{2}\right)
\end{aligned}
$$

where

$$
\begin{aligned}
p=\left\{\begin{array}{ll}
1, & i=1,3,5,7,9, \\
0, & i=2,4,6,8,
\end{array} K_{1}=\right. & -\Omega_{2}^{2} A_{2}+\omega_{02}^{2} A_{2}+\frac{63}{128} \kappa_{9} A_{2}^{9}, \begin{array}{ll}
0 \\
1, & i=2,4,6,8,
\end{array} \\
& +\frac{35}{64} \kappa_{7} A_{2}^{7}+\frac{5}{8} \kappa_{5} A_{2}^{5}+\frac{3}{4} \kappa_{3} A_{2}^{3}, \\
K_{2}= & -\frac{7}{16} \kappa_{8} A_{2}^{8}-\frac{15}{32} \kappa_{6} A_{2}^{6}-\frac{1}{2} \kappa_{4} A_{2}^{4}-\frac{1}{2} A_{2}^{2}, \\
K_{3}= & -\frac{21}{64} \kappa_{9} A_{2}^{9}-\frac{21}{64} \kappa_{7} A_{2}^{7}-\frac{5}{16} \kappa_{5} A_{2}^{5}-\frac{1}{4} \kappa_{3} A_{2}^{3}, \\
K_{4}= & \frac{7}{32} \kappa_{8} A_{2}^{8}+\frac{3}{16} \kappa_{6} A_{2}^{6}+\frac{1}{8} \kappa_{4} A_{2}^{4}, \\
K_{5}= & \frac{9}{64} \kappa_{9} A_{2}^{9}+\frac{7}{64} \kappa_{7} A_{2}^{7}+\frac{1}{16} \kappa_{5} A_{2}^{5}, \\
K_{6}= & -\frac{1}{16} \kappa_{8} A_{2}^{8}-\frac{1}{32} \kappa_{6} A_{2}^{6}, \\
K_{7}= & -\frac{9}{256} \kappa_{9} A_{2}^{9}-\frac{1}{64} \kappa_{7} A_{2}^{7}, \\
K_{8}= & \frac{1}{128} \kappa_{8} A_{2}^{8}, \\
K_{9}= & \frac{1}{256} \kappa_{9} A_{2}^{9} .
\end{aligned}
$$




\section{Inner-Resonance}

The inner-resonance can be expected when one of the following conditions is met:

$$
\begin{aligned}
\Omega_{1} & =\omega_{01}, \\
\Omega_{1} & =\omega_{02}, \\
\Omega_{2} & =\frac{1}{i} \omega_{01}, \quad i=1,2, \ldots, 9, \\
\Omega_{2} & =\frac{1}{i} \omega_{02}, \quad i=1,2, \ldots, 9, \\
\omega_{01} & =\omega_{02} .
\end{aligned}
$$

These conditions should be avoided during the cushioning packaging design procedure.

\section{Conclusion}

The conditions for inner-resonance, which should be avoided in the cushioning packaging design procedure, can be easily obtained using the variational iteration method.

\section{Acknowledgments}

This work was supported by the National Natural Science Foundation of China (Grant no. 51205167), Research Fund of Young Scholars for the Doctoral Program of Higher Education of China (Grant no. 20120093120014), and Fundamental Research Funds for the Central Universities (Grant no. JUSRP51302A).

\section{References}

[1] D.-M. Wang, Z.-W. Wang, and Q.-H. Liao, "Energy absorption diagrams of paper honeycomb sandwich structures," Packaging Technology and Science, vol. 22, no. 2, pp. 63-67, 2009.

[2] Z.-W. Wang and E. Yu Ping, "Mathematical modelling of energy absorption property for paper honeycomb in various ambient humidities," Materials and Design, vol. 31, no. 9, pp. 4321-4328, 2010.

[3] Y. F. Guo and J. H. Zhang, "Shock absorbing characteristics and vibration transmissibility of honeycomb paperboard," Shock and Vibration, vol. 11, no. 5-6, pp. 521-531, 2004.

[4] V. Rouillard, M. A. Sek, and S. Crawford, "The dynamic behaviour of stacked shipping units during transport. Part I: model validation," Packaging Technology and Science, vol. 17, no. 5, pp. 237-247, 2004.

[5] M. A. Garcia-Romeu-Martinez, M. A. Sek, and V. A. CloquellBallester, "Effect of initial pre-compression of corrugated paperboard cushions on shock attenuation characteristics in repetitive impacts," Packaging Technology and Science, vol. 22, no. 6, pp. 323-334, 2009.

[6] J. Wang, F. Duan, J. H. Jiang, and L. X. Lu, "Dropping damage evaluation for a hyperbolic tangent nonlinear system with a critical component," Journal of Vibration and Control, vol. 18, pp. 1417-1421, 2012.

[7] J. Wang, Y. Khan, L. X. Lu, and Z. W. Wang, "Inner resonance of a coupled hyperbolic tangent nonlinear oscillator arising in a packaging system," Applied Mathematics and Computation, vol. 218, no. 15, pp. 7876-7879, 2012.

[8] G. C. Wu, "New trends in the variational iteration method," Communications in Fractional Calculus, vol. 2, no. 2, pp. 59-75, 2011.

[9] G.-C. Wu, "Variational iteration method for $q$-difference equations of second order," Journal of Applied Mathematics, vol. 2012, Article ID 102850, 5 pages, 2012.

[10] H. Kong and L. L. Huang, "Lagrange multipliers of $q$-difference equations of third order," Communications in Fractional Calculus, vol. 3, no. 1, pp. 30-33, 2012.

[11] G. C. Wu and D. Baleanu, "New applications of the variational iteration method-from differential equations to $q$-fractional difference equations," Advances in Difference Equations, vol. 2013, article 21, 2013.

[12] G.-C. Wu and D. Baleanu, "Variational iteration method for the Burgers' flow with fractional derivatives-new Lagrange multipliers," Applied Mathematical Modelling, vol. 37, no. 9, pp. 61836190, 2013.

[13] C. H. Hu, K. L. Zeng, H. R. Feng, Y. Zeng, and J. P. Chen, "Approximate solutions of the ENSO model with fractional derivatives," Communications in Fractional Calculus, vol. 4, no. 1, pp. 11-15, 2013.

[14] H. Jafari and C. M. Khalique, "Homotopy perturbation and variational iteration methods for solving fuzzy differential equations," Communications in Fractional Calculus, vol. 3, no. 1, pp. 38-48, 2012.

[15] F. Fouladi, E. Hosseinzadeh, A. Barari, and G. Domairry, "Highly nonlinear temperature-dependent fin analysis by variational iteration method," Heat Transfer Research, vol. 41, no. 2, pp. 155$165,2010$.

[16] A. Sadighi and D. D. Ganji, "Exact solutions of nonlinear diffusion equations by variational iteration method," Computers and Mathematics with Applications, vol. 54, no. 7-8, pp. 11121121, 2007.

[17] J. H. Jiang and Z. W. Wang, "The research on the vibration characteristics for honeycomb paperboard cushioing package system," Journal of Hubei University of Technology, vol. 21, no. 3, pp. 18-20, 2006.

[18] J.-H. He, "Variational iteration method-a kind of non-linear analytical technique: some examples," International Journal of Non-Linear Mechanics, vol. 34, no. 4, pp. 699-708, 1999.

[19] J.-H. He, "Asymptotic methods for solitary solutions and compactons," Abstract and Applied Analysis, vol. 2012, Article ID 916793, 130 pages, 2012.

[20] L. Xu, "Dynamics of two-strand yarn spinning in forced vibration," Nonlinear Analysis: Theory, Methods \& Applications, vol. 71, no. 12, pp. e827-e829, 2009. 


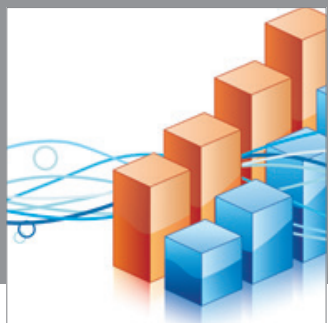

Advances in

Operations Research

mansans

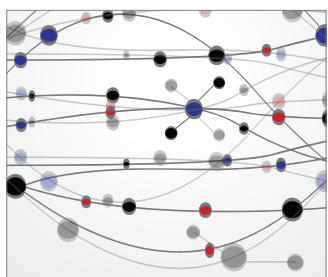

The Scientific World Journal
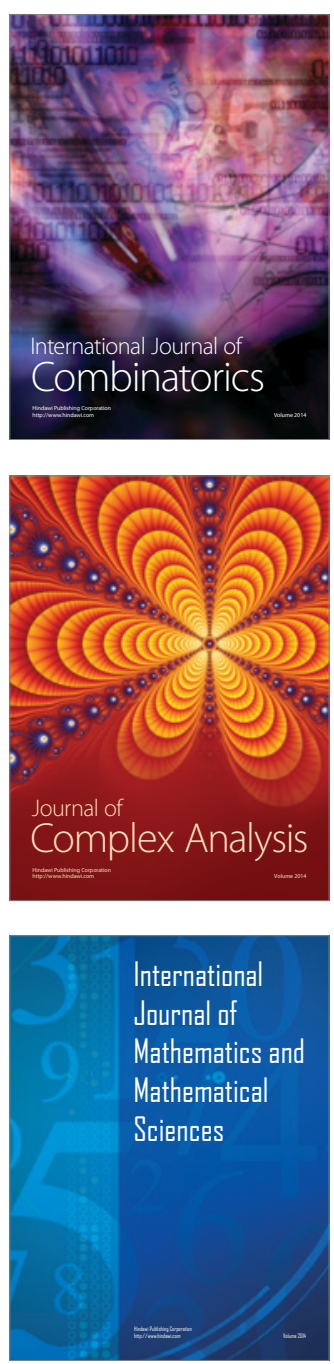
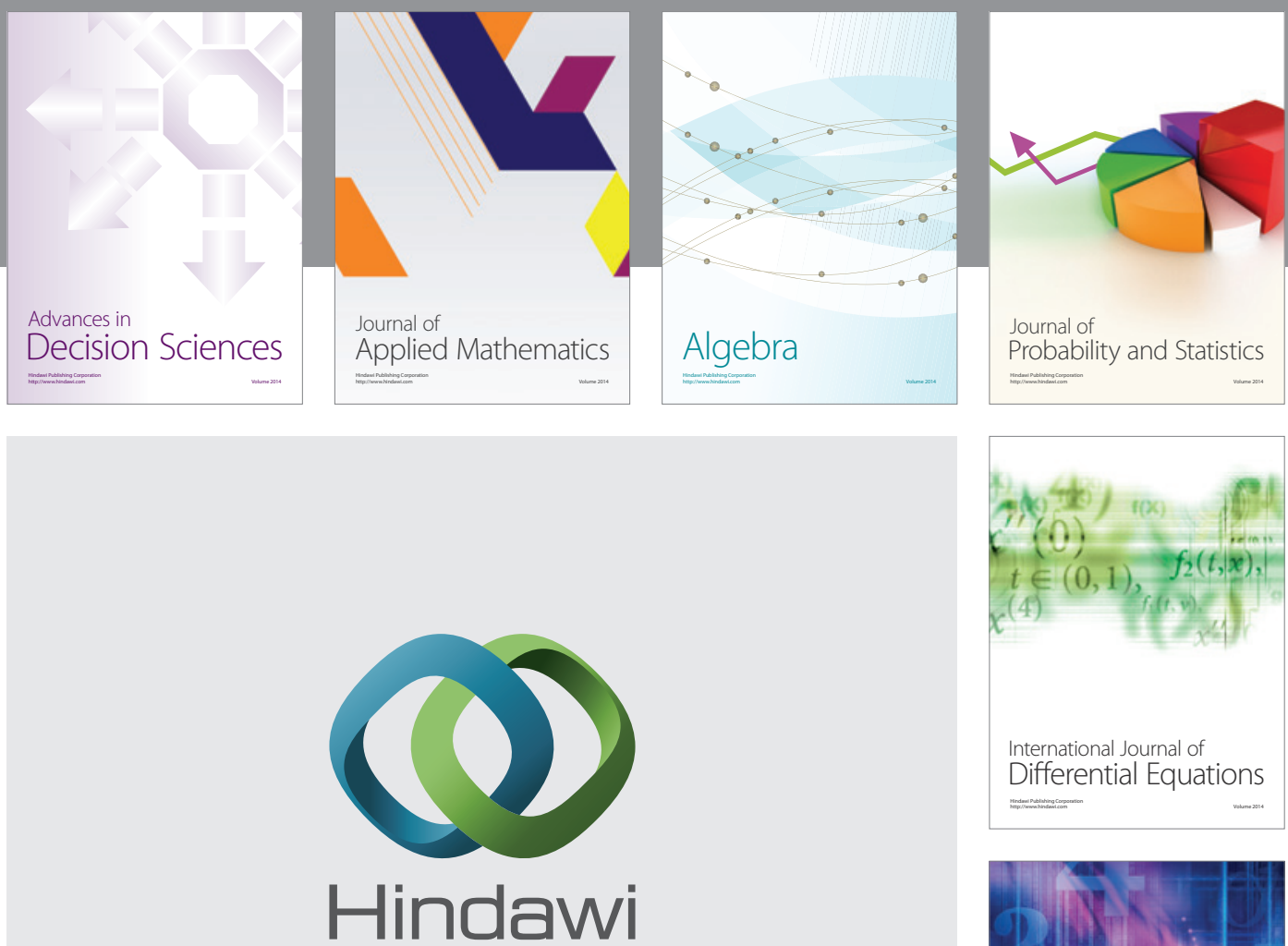

Submit your manuscripts at http://www.hindawi.com
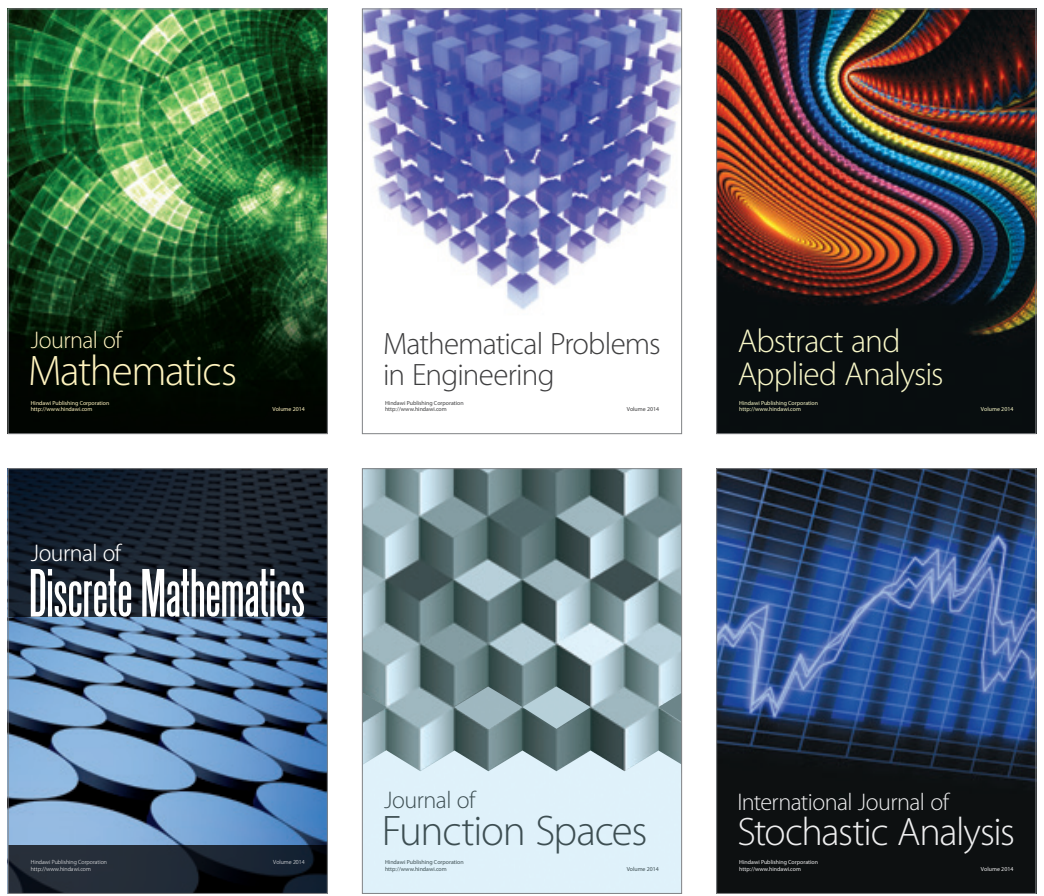

Journal of

Function Spaces

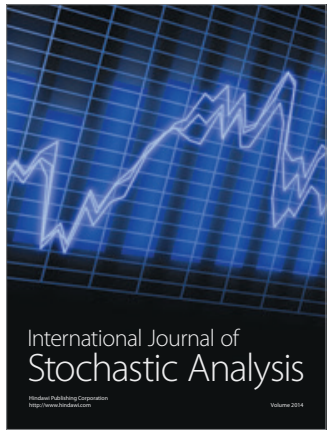

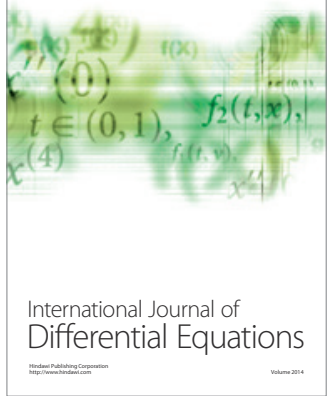
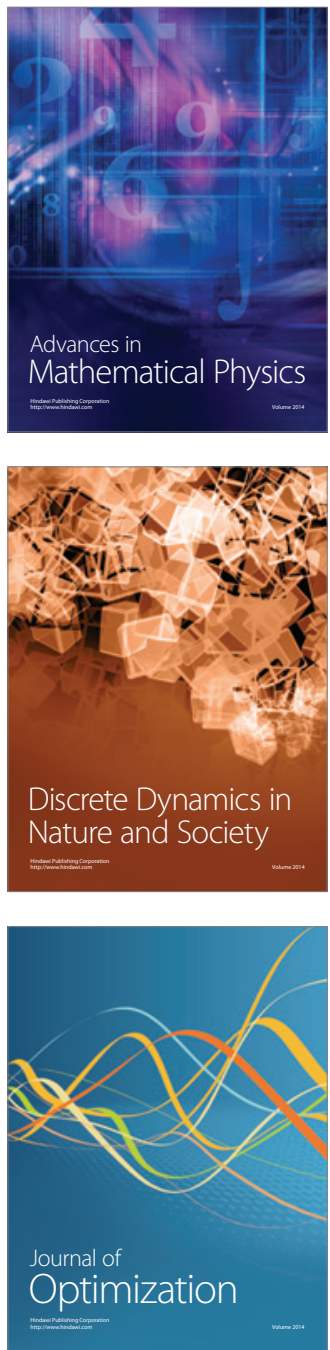